\title{
Roles of the Nanog protein in murine F9 embryonal carcinoma cells and their endoderm-differentiated counterparts
}

\author{
Yanmei Chen ${ }^{1}$, Zhongwei $\mathrm{Du}^{2}$, Zhen Yao ${ }^{1}$ \\ ${ }^{1}$ Laboratory of Molecular and Cell Biology, Institute of Biochemistry and Cell Biology, Shanghai Institutes for Biological Sciences, \\ Chinese Academy of Sciences, Graduate School of the Chinese Academy of Sciences, 320 Yueyang Road, Shanghai 200031, China; \\ ${ }^{2}$ Department of Anatomy, School of Medicine, Waisman Center, Wicell Institute, University of Wisconsin, Madison, WI 53705, USA
}

Nanog is a recently discovered homeodomain transcription factor that sustains the pluripotency of embryonic stem (ES) cells and blocks their differentiation into endoderm. The murine F9 embryonal carcinoma cell line is a well-documented model system for endoderm cell lineage differentiation. Here, we examined the function of Nanog in F9 cell endoderm differentiation. Over-expression of Nanog returns the F9 cells to the early status of ES cells and represses the differentiation of primitive endoderm and parietal endoderm in F9 cells, whereas it has no effect on the differentiation of visceral endoderm. In contrast, the expression of C-terminal domain-truncated Nanog spontaneously promotes endoderm differentiation in F9 cells. These data suggest that Nanog is required to sustain the proper undifferentiated status of F9 cells, and the C-terminal domain of Nanog transduces the most effects in repressing primitive endoderm and parietal endoderm differentiation in F9 cells.

Cell Research (2006) 16:641-650. doi: 10.1038/sj.cr.7310067; published online 6 Jun 2006

Keywords: Nanog, F9 embryonal carcinoma cells, endoderm, differentiation

\section{Introduction}

Murine F9 embryonal carcinoma (EC) cells represent a valuable in vitro model system for studying early endoderm differentiation during mammalian development. The treatment of F9 cell monolayers with retinoic acid (RA), a biologically active molecule related to vitamin A, results in differentiation to the primitive endoderm. When cyclic AMP analogs, such as dibutyryl cyclic cAMP $\left(\mathrm{Bt}_{2} \mathrm{cAMP}\right)$, $\mathrm{PTH}$, and PTHrP, are given to cells concomitant with or after the treatment of RA, further differentiation of F9 cells into the parietal endoderm is induced. When cultured as aggregates, these cells can differentiate into the visceral endoderm $[1,2]$. These differentiation processes are accompanied by morphological changes and expression of

Correspondence: Yanmei Chen

Tel: +86-21-54921368; Fax: +86-21-54921366;

E-mail: chenym@sibs.ac.cn

Received 11 Nov 2005; revised 15 Feb 2006; accepted 20 Mar 2006; published online 6 Jun 2006 several specific markers [3-6].

Murine Nanog is a divergent homeobox protein that shares homology with members of the NK2 homeobox gene family [7, 8]. Nanog cDNA is $2184 \mathrm{bp}$ in length and comprises an open-reading frame that encodes 305 amino acids. Nanog is expressed in embryonic stem (ES) cells, embryonic germ cells, and EC cells, but not in the corresponding differentiated derivatives [7-9]. In the mouse embryonic development, Nanog first appears in the compacted morula and later is restricted to the inner cell mass (ICM) and primordial germ cells [7, 10]. Gain-of-function and loss-of-function studies suggest that Nanog not only plays a crucial role in the maintenance of pluripotency of murine embryos and ES cells but also blocks the differentiation of ICM and ES cells into endoderm [7, 8, 11, 12]. Currently, it is not known how Nanog performs these two distinct and important functions.

Nanog is a multi-domain protein that can be divided into three subdomains according to the location of the homeodomain [7-9]. The N-terminal domain is typical of a transactivation domain, which is rich in serine and threonine. The 60-residue homeodomain is assumed to be 
responsible for recognition, binding of a sequence-specific DNA motif, and protein-protein interactions, as demonstrated for Oct-4 [13]. The C-terminal domain has a tryptophan repeat motif, in which Trp-X-X-X is repeated 10 times in tandem. This motif may represent a novel domain with unknown structural and functional properties [7-9]. Pan and colleagues have reported Nanog as a transcription activator that is capable of transactivating a reporter plasmid bearing its cognate binding sites. Both the N-terminal domain and $\mathrm{C}$-terminal domain contain transactivation activities, but the $\mathrm{C}$-terminal domain is a much stronger activator $[14,15]$. However, it is not clear how the two domains of the Nanog protein contribute to its function. To determine which domain of the Nanog protein is responsible for the blocking of endoderm differentiation, we over-expressed different truncated Nanog in F9 cells and examined the influence on endoderm differentiation.

\section{Materials and Methods}

\section{Cell culture and differentiation}

Murine F9 cells were cultured on $0.1 \%$ gelatin-coated dishes in Dulbecco's modified Eagle's medium that was supplemented with $10 \%$ fetal calf serum. Differentiation to primitive endoderm was performed by culturing the cells in monolayer in the presence of 1 $\mu \mathrm{m}$ of all-trans-RA (Sigma Chemical Co., St Louis, MO, USA) for 3 days. To obtain parietal endoderm, this treatment was followed by $1 \mathrm{mM} \mathrm{Bt}{ }_{2}$ AMP (Sigma) for 2 days [1,2]. To generate visceral endoderm, the F9 cells were grown in suspension as aggregates in Petri dishes with $50 \mathrm{nM}$ RA for 7 days $[1,2,16]$. On the 7 th day, the embryoid bodies were re-plated to obtain monolayer outgrowths of visceral endoderm cells. Morphological changes and outgrowth were monitored photo-microscopically. Differentiated phenotypes were confirmed by Northern blot analysis using appropriate markers.

\section{Plasmids and stable transfection}

Expression plasmids that contained either full-length or partiallength Nanog cDNAs were constructed by insertion into the HAtagged vector pCBA-hrGFP (kindly provided by Sangmi Chung)
[17]. All of the Nanog cDNAs were PCR-amplified by using the high-fidelity Pyrobest DNA polymerase (Takara, Kyoto, Japan), cloned, and sequenced. The following primer pairs were used to amplify the full-length and partial-length of Nanog cDNAs: for the $\mathrm{N}$-terminal and homeodomain portion of Nanog cDNA (designated NH), NHS (sense) 5'-CAT GTC GAC TCA GTG TGG GTC TTC CT- $3^{\prime}$ and NHA (antisense) 5'-CGC CTC GAG CTG CCA CCG CTT GCA-3'; for the homeodomain and C-terminal portion of Nanog cDNA (designated CH), CHS (sense) 5'-GCG GTC GAC TCA AGC AGA AGA T-3' and CHA (antisense) $5^{\prime}$-CGC CTC GAG TAT TTC ACC TGG T-3'. For the full-length Nanog cDNA, NHS and CHA were used. The F9 cells were transfected with Lipofectamine 2000 (Invitrogen, Carlsbad, CA, USA) according to the manufacturer's instructions, and the transfectants were selected with $800 \mu \mathrm{g} / \mathrm{ml}$ G418 for 15 days. F9 cells transfected with the empty vector were used as the control.

\section{Immunocytochemistry}

F9 cells were seeded onto slides that were coated with $0.1 \%$ gelatin, and fixed first in $4 \%$ paraformaldehyde for $15 \mathrm{~min}$, and then in methanol for $5 \mathrm{~min}$ at room temperature. The cells were washed three times in ice-cold PBS and permeabilized with $0.1 \%$ Triton/PBS for $10 \mathrm{~min}$. Then, cells were blocked with $1 \% \mathrm{BSA} / \mathrm{PBS}$ for $30 \mathrm{~min}$ at room temperature and incubated in the primary antibody, MC-480 supernatant, which contained anti-SSEA-1 antibody (purchased from DSHB, University of Iowa), at $4{ }^{\circ} \mathrm{C}$ overnight. FITC-conjugated goat anti-mouse IgG (Santa-Cruz Biotechnology, Santa Cruz, CA, USA) was used as the secondary antibody. Reactions to anti-Oct-4 antibody (Santa-Cruz Biotechnology) and anti-Gata-4 antibody (Santa-Cruz Biotechnology) were performed in the same way.

\section{Northern blotting}

Total RNA was prepared using the TRIZOL reagent (Invitrogen). From each sample, $30 \mu \mathrm{g}$ RNA was separated on a $1 \%$ agarose formaldehyde denaturing gel and transferred to a nylon membrane. Hybridization was performed at $42{ }^{\circ} \mathrm{C}$ overnight using the ${ }^{32} \mathrm{P}$-labeled probes in hybridization solution containing formamide. The membranes were washed twice with $0.2 \times \mathrm{SSC}, 0.1 \% \mathrm{SDS}$ at room temperature for $30 \mathrm{~min}$, twice with $0.2 \times \mathrm{SSC}, 0.1 \% \mathrm{SDS}$ at $42^{\circ} \mathrm{C}$ for $30 \mathrm{~min}$, and then exposed to X-ray film at $-80^{\circ} \mathrm{C}$ with an intensifying screen for $72 \mathrm{~h}$. The same blot was hybridized with ${ }^{32} \mathrm{P}$-labeled mouse GAPDH cDNA as the control probe.

Table 1 List of gene-specific primer sets

\begin{tabular}{llll}
\hline Gene & Forward primer & Reverse primer & Size of product (bp) \\
\hline Oct-4 & acctggctaagcttccaagg & gtgatcctcttctgcttcag & 358 \\
Gata-6 & gcaatgcatgcggtctctac & ctcttggtagcaccagctca & 571 \\
Gata-4 & gcctgtatgtaatgcctgcg & ccgagcaggaatttgaagagg & 469 \\
Hnf1 $\beta$ & gaaagcaacgggagatcctccgac & cctccactaaggcctccctctcttcc & 279 \\
LamininB1 & ctgttctgaaagtgaatgtggtggcccc & gtttaatcgccttctctgctgcaacctg & 628 \\
Dab2 & ggcaacaggctgaaccattagt & ttggtgtcgatttcagagtttagat & 283 \\
Afp & tcgtattccaacaggagg & aggctttgcttcaccag & 174 \\
Ttr & agtcctggatgctgtccgag & ttcctgagctgctaacacgg & 440 \\
GAPDH & accacagtccatgccatcac & tccaccacctgttgctgta & 452 \\
\hline
\end{tabular}




\section{Western blotting}

Whole-cell protein extracts were fractioned by SDS-PAGE and immunoblotted onto nitrocellulose. The blots were incubated in a 1:10 000 dilution of the primary anti-HA monoclonal antibody (Sigma) at room temperature for $1 \mathrm{~h}$, and then washed with $0.1 \%$ Tween-20/PBS. Horseradish peroxidase-conjugated goat anti-mouse secondary antibodies (Santa Cruz Biotechnology) were used at a 1:2000 dilution and the blots were developed using the Supersignal West Pico Trial Kit (Pierce, Rockford, IL, USA).

\section{$R T-P C R$}

Total RNA was prepared using the TRIZOL reagent (Invitrogen) and reverse-transcribed with oligo-(dT) primer (Promega) and StrataScript Reverse Transcriptase (Stratagene, La Jolla, CA, USA). PCR was carried out with ExTaq (Takara). The PCR products were fractionated by electrophoresis on a $2 \%$ agarose gel. The primer sets are listed in Table 1 [18].

\section{Results}

Expression of Nanog during F9 cell endoderm differentiation

Nanog has been reported to express in F9 cell lines [7]. To study the effect of Nanog on endoderm differentiation, we first examined its expression during endoderm differentiation of F9 cells by Northern blot. Nanog was

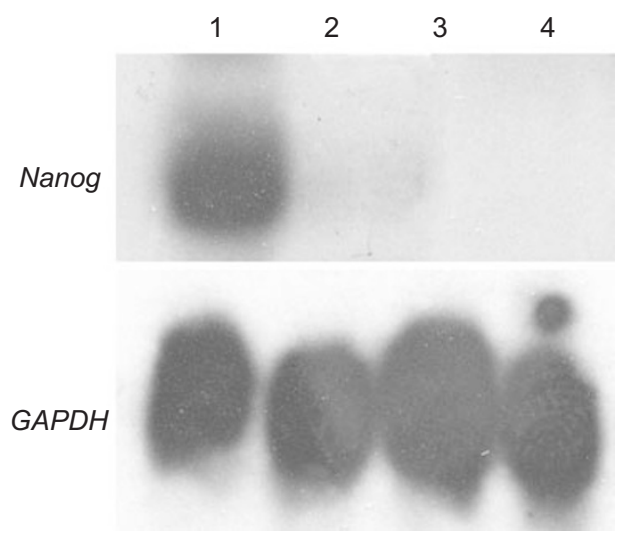

Figure 1 Expression of Nanog gene during endoderm differentiation of F9 cells. RNA was isolated from untreated F9 cells (lane 1), or from cells differentiate into primitive endoderm (lane 2), parietal endoderm (lane 3), and visceral endoderm (lane 4).

abundantly expressed in undifferentiated F9 cells, but its expression was remarkably reduced and even undetectable in the primitive endoderm, parietal endoderm, and visceral endoderm cells upon differentiation (Figure 1).

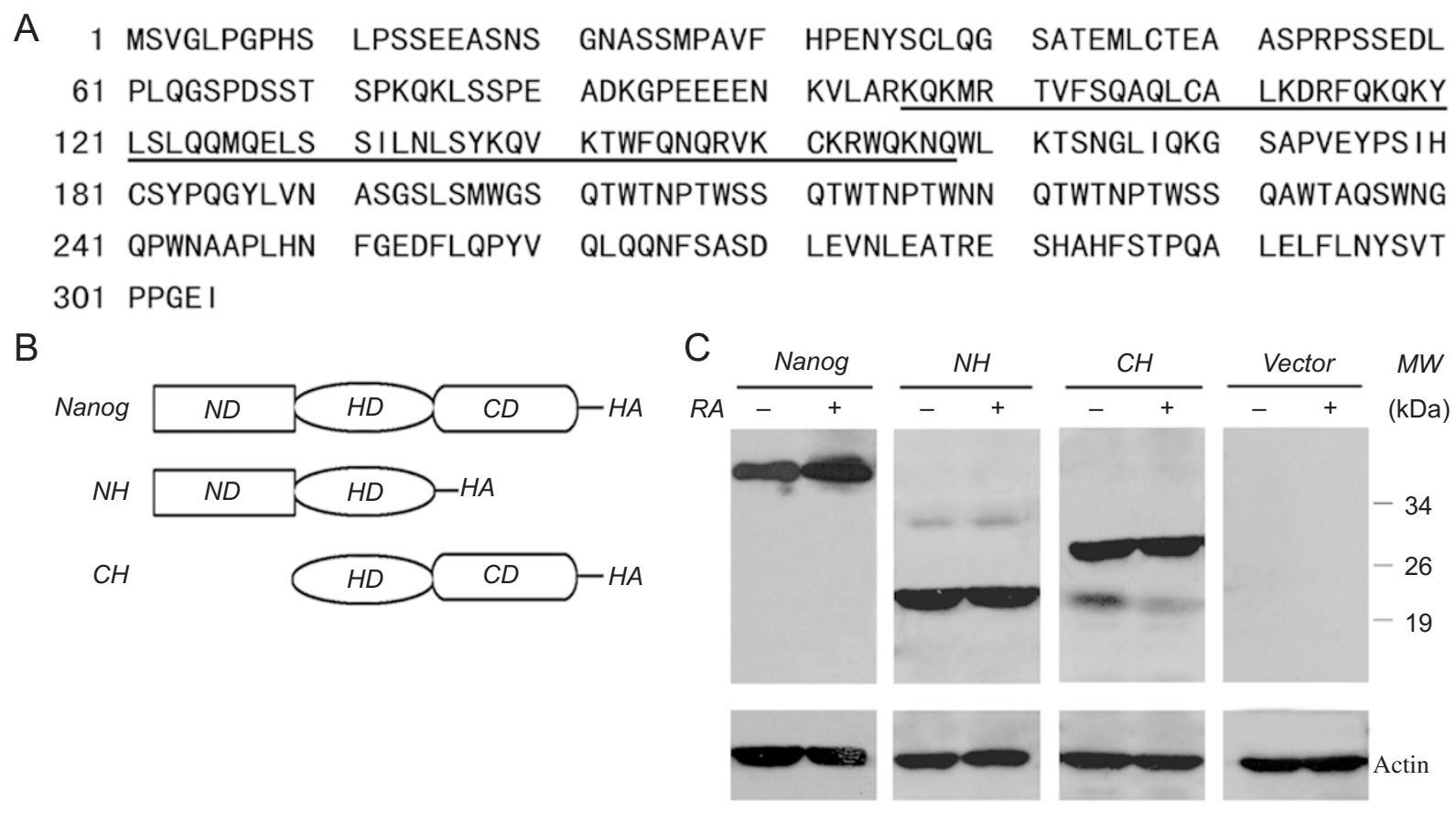

Figure 2 Characterization of different Nanog F9 cell clones. (A) Amino acid sequence of Nanog protein. The homeodomain was underlined. (B) Schematic illustrations of three Nanog cDNA expression constructs. The HA tag is located at the C-terminal end of the constructs. ND: N-terminal domain; HD: homeodomain; and CD: C-terminal domain. (C) Western blotting analysis of different Nanog constructs expressions in four F9 cell clones before and after RA addition with anti-HA monoclonal antibody. 


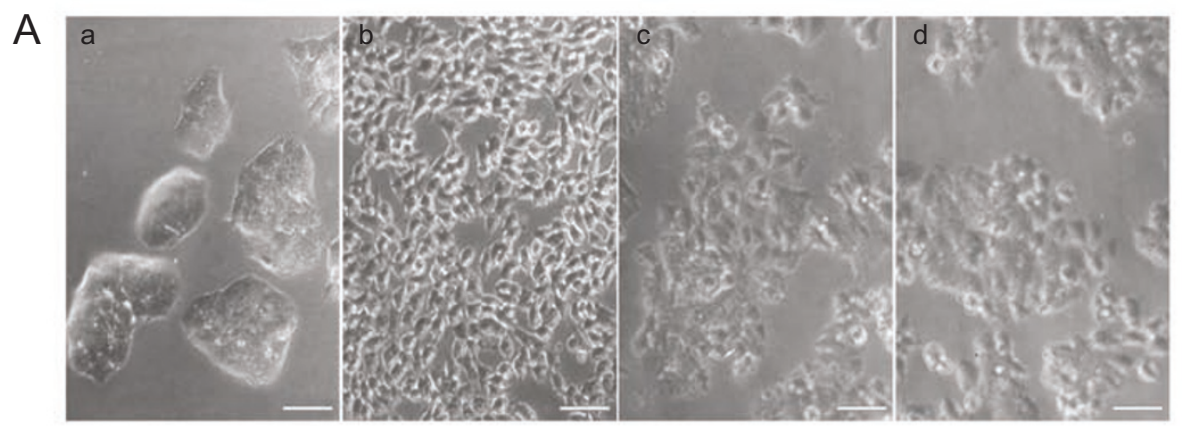

B
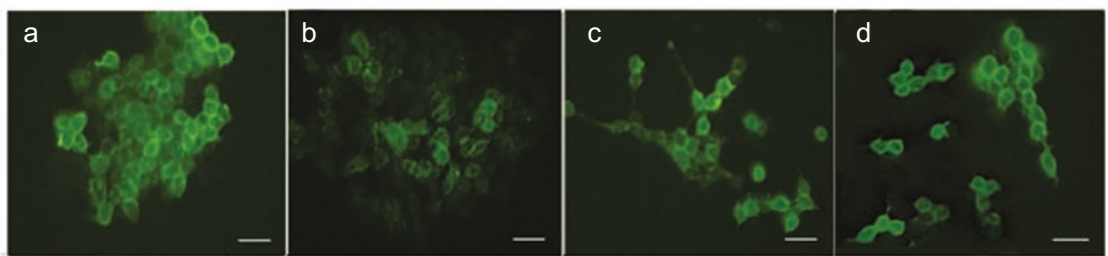

SSEA-1
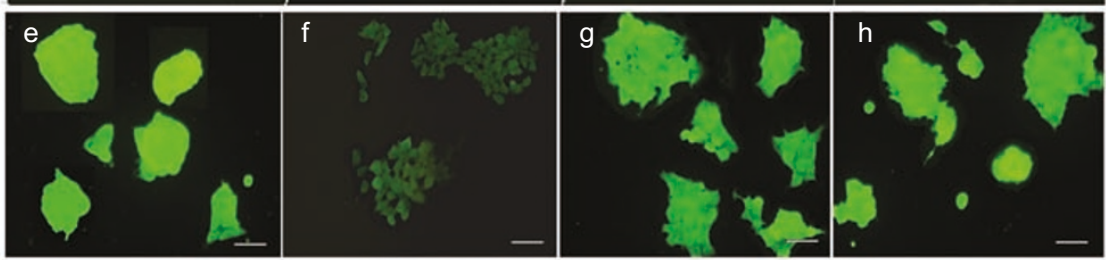

Oct-4
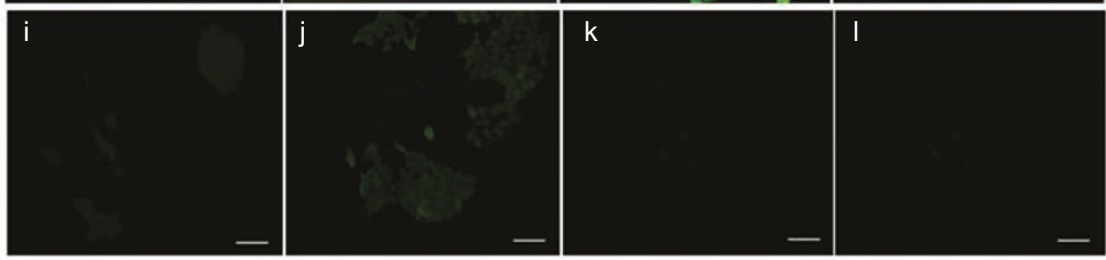

Gata-4

C

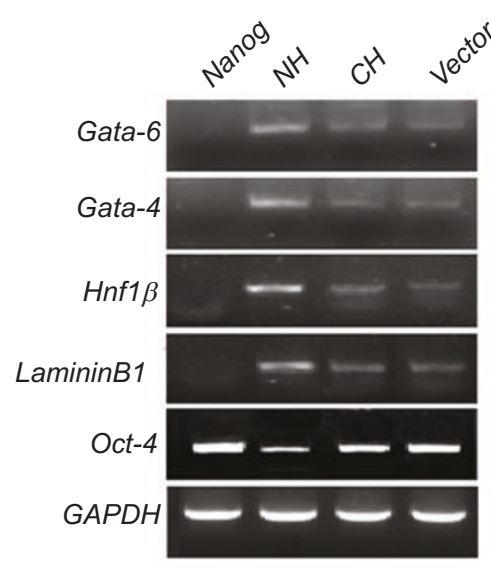

Figure 3 Comparison of morphology, SSEA-1, Oct-4, and Gata-4 staining, and several marker gene expressions between four undifferentiated F9 cell clones. (A) Phase-contrast images of F9-Nanog cells (a), F9-NH cells (b), F9-CH cells (c), and F9-Vector cells (d). (B) Immunofluorescence analysis of SSEA-1, Oct-4, and Gata-4 expressions in F9-Nanog cells (a, e, i), F9-NH cells (b, f, j), F9-CH cells (c, g, k), and F9-Vector cells (d, h, 1). (C) RT-PCR analysis of several marker genes in four F9 cell clones. Bar $=100 \mu \mathrm{m}$.

Over-expression of Nanog returns the F9 cells to the early status of ES cells

To investigate the contributions of the different domain of the Nanog protein to the prevention of endoderm differentiation, we constructed a series of expression plasmids that were driven by the $\beta$-actin promoter, as illustrated in

Figure 2B. This promoter can drive high-level expression of the inserted gene, and the expression level is not affected by the induction of RA [17, 19]. Expression constructs containing the intact Nanog protein or lacked either Nterminal domain or C-terminal domain were generated and transfected into the F9 cells to generate stable cell lines (F9- 
A

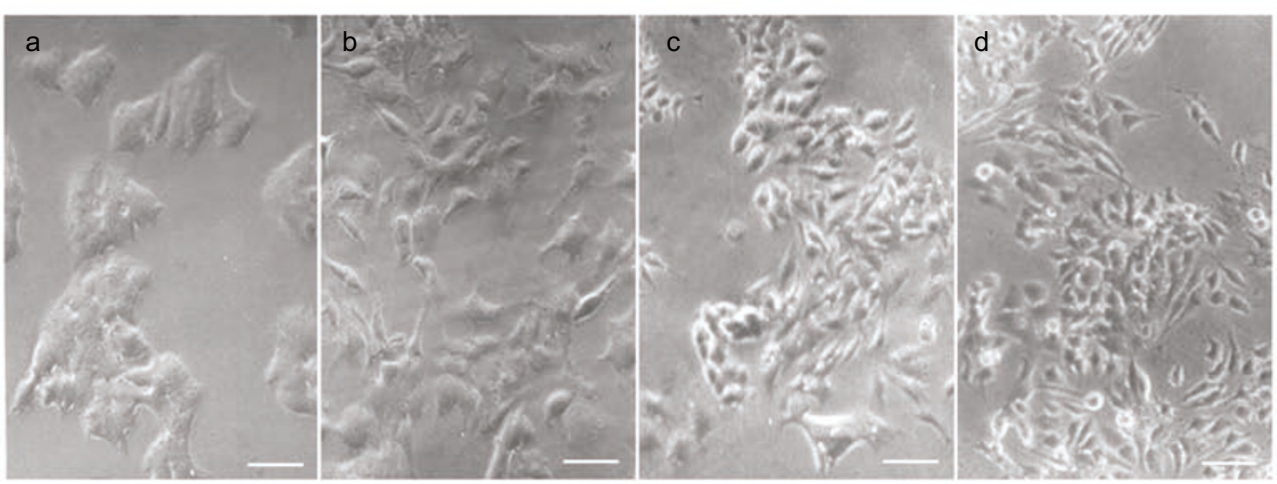

B

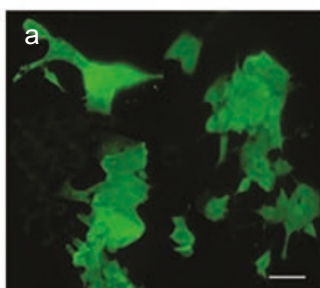

\section{b}
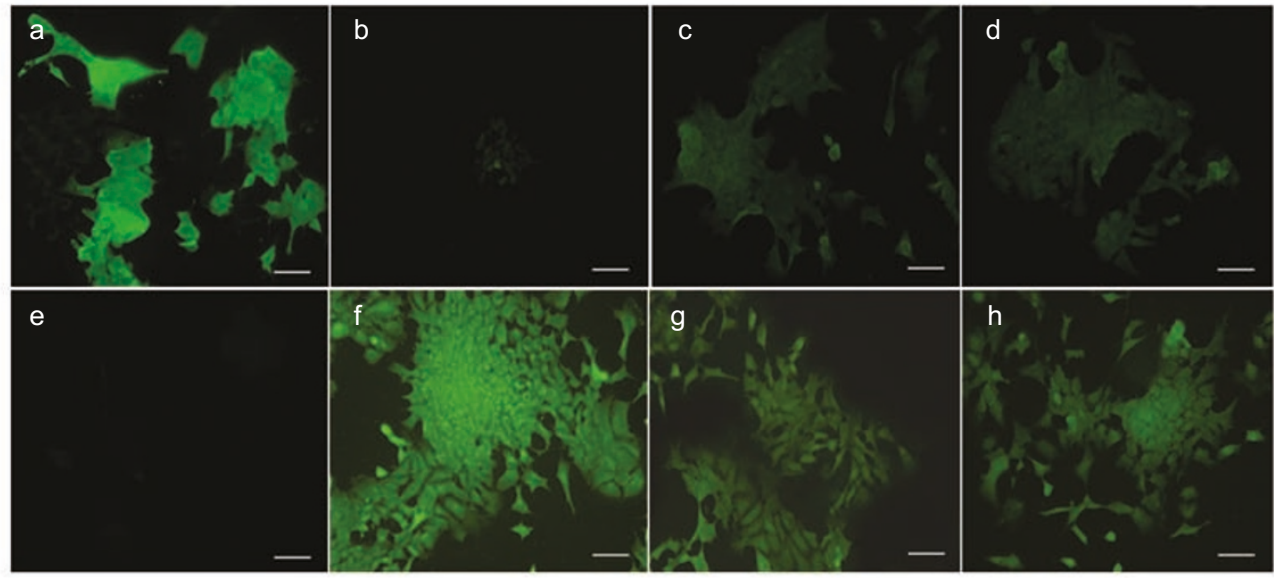

Oct-4

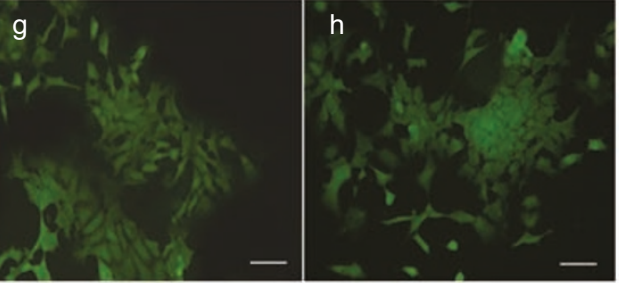

Gata-4

C

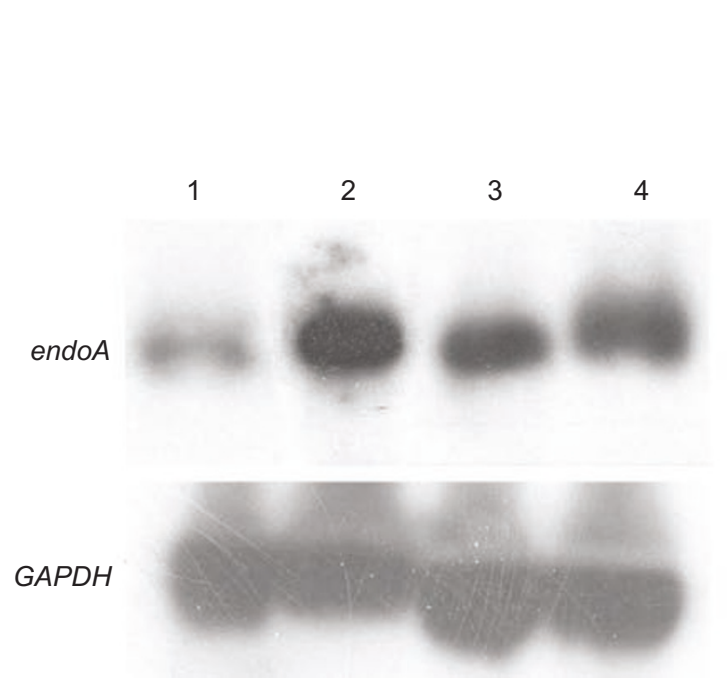

D

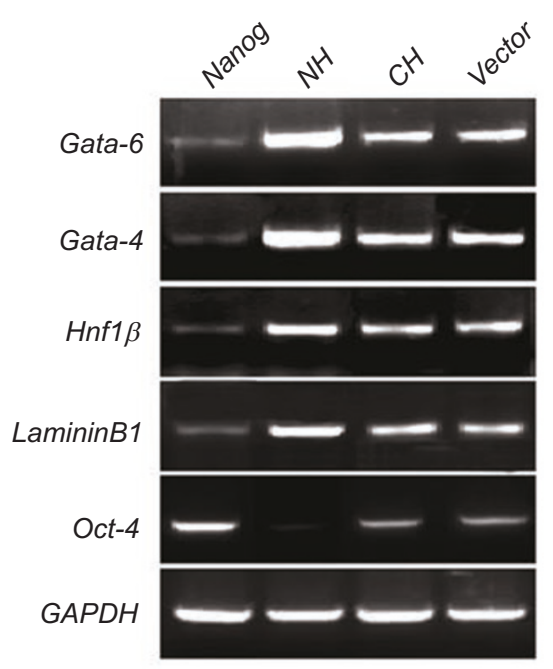

Figure 4 Comparison of morphology, endoA expression and several marker gene expression patterns between four F9 cells clones during primitive endoderm differentiation. (A) Phase-contrast images of differentiated F9-Nanog cells (a), F9-NH cells (b), F9-CH cells (c), and F9-Vector cells (d). (B) Immunofluorescence analysis of Oct-4 and Gata-4 expressions in F9-Nanog cells (a, e), F9-NH cells (b, f), F9-CH cells (c, g), and F9-Vector cells (d, h). (C) Analysis of the expression of primitive endoderm marker by Northern blot. Lanes: 1, F9-Nanog cells; 2, F9-NH cells; 3, F9-CH cells; and 4, F9-Vector cells. (D) RT-PCR analysis of several marker genes in four F9 cells clones. Bar $=100 \mu \mathrm{m}$. 
Nanog cells, F9-CH cells, and F9-NH cells, respectively). By using the anti-HA monoclonal antibody, the expression of the different transgene products was ascertained for each transfectant by Western blotting. Each transfectant showed a strongly hybridizing band of the predictive size before and after RA induction (Figure 2C), whereas F9 cells that contained the empty vector alone (F9-Vector cells) did not exhibit any hybridizing band.

After the establishment of the different stable F9 cell lines, we found that the over-expression of Nanog caused the F9 cells to adopt a tightly packed rounded shape rather than growing as an even monolayer as normal F9-Vector cells. These compact colonies resembled ES cells cultured on feeder layers (Figure 3A, a, d). Immunofluorescence analysis showed that the F9-Nanog cells reacted strongly to the stem cell marker SSEA-1 (Figure 3B, a). We also examined the expression of stem cell-specific and endoderm-specific genes by RT-PCR. Faint bands were detected for Gata-6, Gata-4, Hnf1 $\beta$, and Laminin B1 in the F9-Vector cells but not in the F9-Nanog cells. Oct-4, which is a stem cell marker, was strongly expressed in F9-Nanog cells, whereas its expression was weaker in F9-Vector cells (Figure. 3B, e, h and 3C). These data show for the first time that the over-expression of Nanog can return the F9 cells to the early status of ES cells.

\section{Expression of truncated Nanog spontaneously promotes F9 cells endoderm differentiation}

The F9-NH cells displayed a morphology that differed strikingly from the F9-Vector and F9-CH cells (Figure $3 \mathrm{~A}, \mathrm{~b}, \mathrm{~d}, \mathrm{c})$. The F9-NH cells grew as isolated cells that resembled slightly differentiated F9 cells (compare Figure $3 \mathrm{~A}, \mathrm{~b}$ with Figure 4A, d). In contrast, the morphology of the F9-CH cells was the same as that of the F9-Vector cells (Figure 3A, c, d). In contrast to the strong expression of SSEA-1 or Oct-4 in F9-Nanog cells, the reactivity of the F9-NH cells to the anti-SSEA-1 or anti-Oct-4 antibody was weaker than that of the control transfectant (Figure $3 \mathrm{~B}, \mathrm{~b}, \mathrm{~d}$ and $3 \mathrm{~B}, \mathrm{f}, \mathrm{h})$. Furthermore, the expression levels of endoderm marker genes were higher in F9-NH cells than in F9-Vector cells, whereas the expression of Oct-4 was lower (Figure 3C). The F9-CH cells showed no differences, as compared to the F9-Vector cells (Figure 3B, c, d, $\mathrm{g}, \mathrm{h}, \mathrm{k}, \mathrm{l}$ and $3 \mathrm{C}$ ). These results suggest that $\mathrm{F} 9-\mathrm{NH}$ cells differentiate spontaneously into primitive endoderm cells, and C-terminal domain-truncated Nanog loses the function
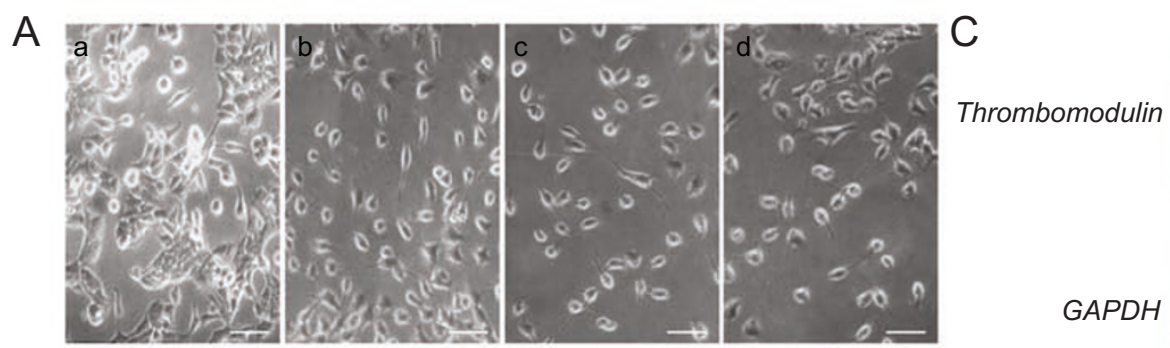

B

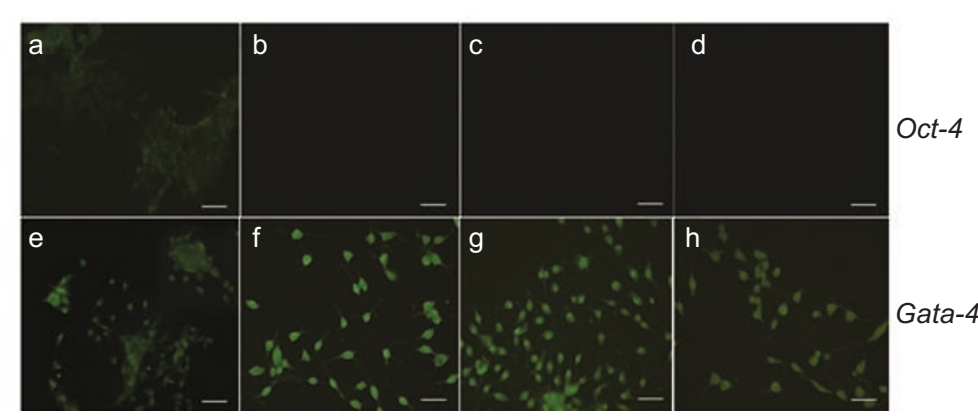

Figure 5 Comparison of morphology, Thrombomodulin expression, and several marker gene expression patterns between four F9 cells clones during parietal endoderm differentiation. (A) Phase-contrast images of differentiated F9-Nanog cells (a), F9-NH cells (b), F9-CH cells (c), and F9-Vector cells (d). (B) Immunofluorescence analysis of Oct-4 and Gata-4 expressions in F9-Nanog cells (a, e), F9-NH cells (b, f), F9-CH cells (c, g), and F9-Vector cells (d, h). (C) Analysis of the expression of parietal endoderm marker by Northern blot. Lanes: 1, F9-Nanog cells; 2, F9-NH cells; 3, F9-CH cells; and 4, F9-Vector cells. (D) RT-PCR analysis of several marker genes in four F9 cells clones. Bar $=100 \mu \mathrm{m}$. 
to repress endoderm differentiation of F9 cells.

Forced expression of Nanog can inhibit primitive endoderm and parietal endoderm differentiation of $\mathrm{F} 9$ cells

To investigate the roles of the N-terminal domain-truncated and C-terminal domain-truncated Nanog protein in primitive endoderm differentiation, the cells were evaluated by morphology analysis and the expression patterns of marker genes were also examined. When induced by RA, the F9-Vector cells and F9-CH cells underwent extensive differentiation and showed an enlarged and flattened mor- phology that is characteristic of primitive endoderm cells [1] (Figure 4A, c, d), whereas most of the F9-Nanog cells remained morphologically undifferentiated (Figure 4A, a). After treatment with RA for 3 days, the F9-NH cells were morphologically indistinguishable from the F9-Vector cells (Figure 4A, b, d). However, Northern blot analysis revealed that the expression of endoA, a specific marker for primitive endoderm [3, 4], was stronger in F9-NH cells and weaker in F9-Nanog cells compared with the F9-CH and F9-Vector cells (Figure $4 \mathrm{C}$ ). In addition, the levels of the other endoderm-specific genes like Gata-6, Gata-4, Hnfl $\beta$, and
A

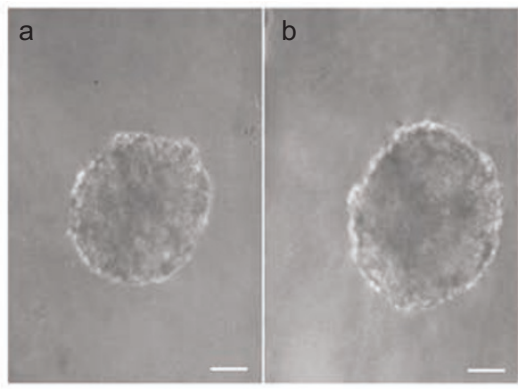

B

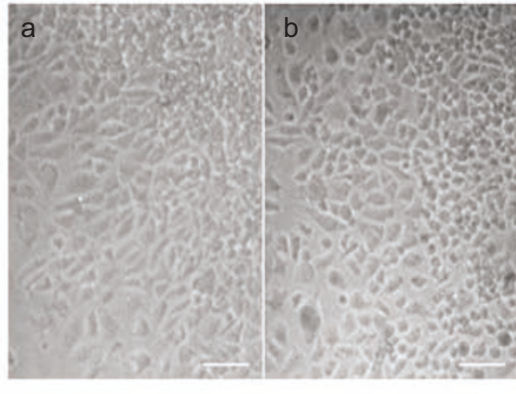

C

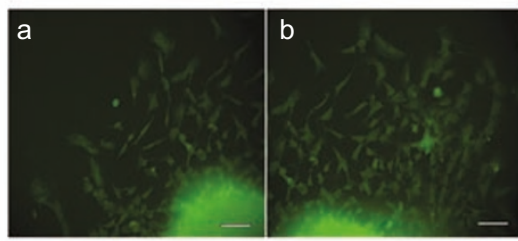

D

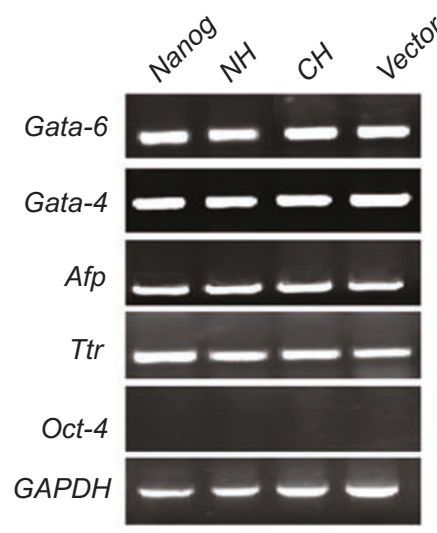

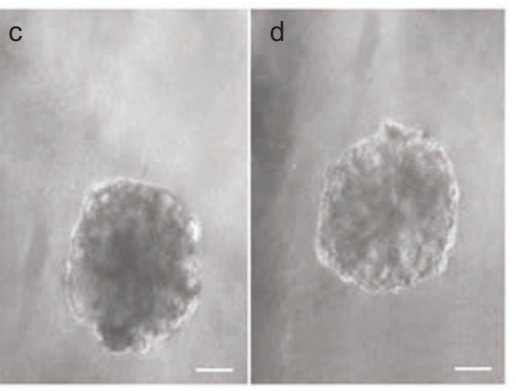

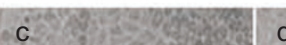

d
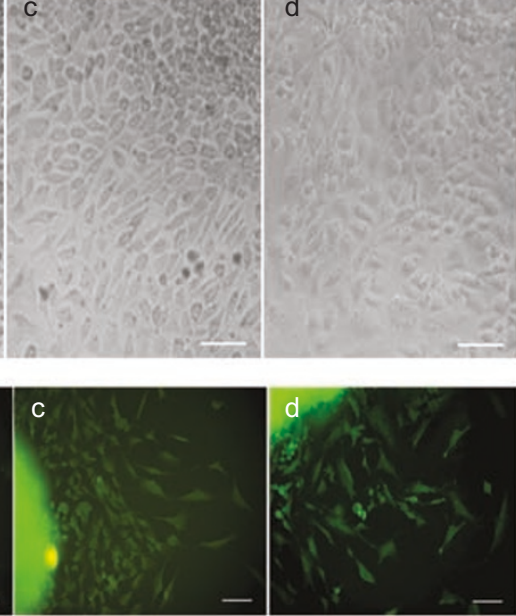

Afp
Figure 6 Comparison of embryoid body formation and re-plated outgrowth morphology and marker gene expression patterns between four F9 cells clones during visceral endoderm differentiation. (A) Phase-contrast images of F9-Nanog cells (a), F9-NH cells (b), F9-CH cells (c), and F9-Vector cells (d) embryoid body. (B) Phase-contrast images of F9-Nanog cells (a), F9-NH cells (b), F9-CH cells (c), and F9-Vector cells (d) embryoid body outgrowths after re-plating. (C) Immunofluorescence analysis of Afp expression in F9-Nanog cells (a), F9-NH cells (b), F9-CH cells (c), and F9-Vector cells (d). (D) RT-PCR analysis of several marker genes in four F9 cells clones. Bar $=100 \mu \mathrm{m}$. 
LamininB1, were low in F9-Nanog cells and high in F9-NH cells. In contrast, the expression of Oct-4 was maintained in the F9-Nanog cells and totally lost in the F9-NH cells (Figure 4D). Immunofluorescence analysis showed the same results (Figure 4B). These data strongly suggest that Nanog can inhibit the primitive endoderm differentiation of F9 cells, whereas C-terminal domain-truncated Nanog can promote this differentiation.

We also examined the roles of the N-terminal domaintruncated and C-terminal domain-truncated Nanog in the further differentiation of F9 cells into parietal endoderm. After exposure to RA for 5 days, and treatment with $1 \mathrm{mM}$ $\mathrm{Bt}_{2} \mathrm{cAMP}$ for the last 2 days, some of the F9-Nanog cells remained undifferentiated, whereas the other three stabletransfected cell lines differentiated fully (Figure 5A). Immunofluorescence analysis showed the remaining reactivity of the F9-Nanog cells to the anti-Oct-4 antibody and a little weak reactivity to the anti-Gata-4 antibody (Figure 5B, a, e). At this time point, there were no differences between the F9-CH, F9-NH, and F9-Vector cells. The expression of Thromobodulin, a unique and definitive marker of parietal endoderm [5], was slightly lower in the F9-Nanog cells than that in the other three cell lines (Figure 5C). The inhibition of Gata-6, Gata-4, Hnfl $\beta$, and LamininB1 expression persisted in the F9-Nanog cells and it is only in this cell line that the expression of Oct-4 could be detected (Figure 5D). These data indicate that Nanog can inhibit the parietal endoderm differentiation of F9 cells.

Forced expression of Nanog has no effect on visceral endoderm differentiation of F9 cells induced by long-term exposure to $R A$

F9 cells cultured as aggregates in $50 \mathrm{nM}$ RA for 7 days differentiate into visceral endoderm cells [16]. The visceral endoderm specifically synthesizes $A f p$, which is not found in the parietal endoderm [6]. When induced to become visceral endoderm, the F9-Nanog, F9-NH, and F9-CH cells showed the same morphology as the F9-Vector cells, as evaluated by the embryoid bodies or the monolayer outgrowths of the embryoid body (Figure 6A and 6B). Immunofluorescence analysis and RT-PCR revealed that there was no difference between the expression of the two specific visceral endoderm markers Afp and transthyretin (Ttr) [20], as well as other endoderm-specific genes (Figure $6 \mathrm{C}$ and $6 \mathrm{D}$ ), which suggests that the forced expression of Nanog has no effect on the visceral endoderm differentiation of F9 cells.

\section{Discussion}

The second differentiation event in mammalian embryogenesis is characterized by the appearance of an epithelial layer on the free surface of the ICM, the primitive endoderm, which subsequently differentiates only into the major fetal components of the yolk sac, the parietal endoderm, and visceral endoderm [21]. The gene regulation network that is involved in these processes is poorly understood. However, several recent reports have indicated that Nanog functions as a suppressor in this endoderm differentiation $[7,8,11,12,22]$. Consistent with these reports, our study found that the over-expression of Nanog repressed primitive endoderm and parietal endoderm differentiation, and inhibition of endogenous Nanog by over-expression of Cterminal domain-truncated Nanog spontaneously promoted the endoderm differentiation.

Nanog is also reported to play critical role in maintaining ES cell pluripotency. The expression of Nanog is restricted in the ICM and primitive ectoderm, but excluded from the primitive endoderm in mouse early embryo [7, 9]. Murine ES cells and F9 cells resemble certain cells of preimplantation embryos, i.e., ICM and primitive ectoderm, respectively $[1,2,21]$. In this study, when Nanog was over-expressed in F9 cells, it leaded the F9 cells to adopt the cellular morphology similar as ES cells. Zhang et al. [23] recently reported that NIH3T3 cells transfected with Nanog gene were round-shaped in culture. Our result is consistent with it. They also showed that the expression of exogenous Nanog gene promoted NIH3T3 cells to enter into the $\mathrm{S}$ phase of cell cycle and increased the cell proliferation. Thus, Nanog might function in a similar manner in our F9 cells as in ES cells, which need additional studies. Furthermore, over-expression of Nanog up-regulated the expression of ES cell markers Oct-4 and SSEA-1 and down-regulated the expression of differentiated endoderm genes Gata-6, Gata-4, Hnf1 $\beta$, and LamininB1, suggesting that elevated Nanog returned the F9 cells to the early status of ES cells. However, inhibition of endogenous Nanog by over-expression of C-terminal domain-truncated Nanog spontaneously promoted endoderm differentiation. These data suggest that the expression level of Nanog appears to be critical for the maintenance of F9 cells status, with any increase or decrease triggering dedifferentiation or differentiation, respectively. Niwa et al. [24] once reported the similar model of Oct-4 with quantitative level determining the different cell fates of ES cells. Over-expression of Nanog up-regulated ES cell gene Oct-4 and down-regulated primitive endoderm genes, which is consistent with the recent report that transcription factors Oct-4, Nanog, and Sox-2 collaborate to form regulatory circuitry consisting of autoregulatory and feedforward loops and co-occupy a substantial portion of their target genes [25]. Thus, the combination of both Nanog and Oct-4 leads to renewal and pluripotency of ES cells, and then they sequentially repress the differentiation of ES cells into extra-embryonic 
lineages - first to trophectoderm (Oct-4) then to primitive endoderm (Nanog).

As a transcription factor, Nanog may maintain stem cell pluripotency and prevent endoderm differentiation by switching on or off its target genes. In our study, the overexpression of Nanog down-regulated endoderm marker gene, Gata-6, as reported to be the direct target gene of Nanog previously [11, 12, 22]. Similar to our results, Nanog null ES cells spontaneously differentiated into parietal endoderm and visceral endoderm [8], in which Gata-6 and Gata-4 were expressed at high levels [18]. This suggests that Nanog may act to repress endoderm differentiation by inhibiting the expression of Gata-6, then its downstream gene Gata-4 [26, 27]. The deletion of either C-terminal domain or N-terminal domain of Nanog ablated its inhibition function, indicating that the two subdomains are both required in repressing endoderm differentiation, although the activity of these two subdomains is not identical in F9 cells. The over-expression of C-terminal domain-truncated Nanog resulted in the spontaneous differentiation and promoted differentiation toward primitive endoderm, which is consistent with the report of Pan and Pei $[14,15]$ that the $\mathrm{C}$-terminal domain is a much stronger activator than its $\mathrm{N}$-terminal domain counterpart.

In summary, we demonstrate for the first time that overexpression of Nanog can return the F9 cells to the early status of ES cells, and repress the primitive endoderm and parietal endoderm differentiation, but not their visceral endoderm differentiation. And the two subdomains, C-terminal domain and $\mathrm{N}$-terminal domain, both have indispensable function in this endoderm repression, but $\mathrm{C}$-terminal domain of Nanog transduces the most effect.

\section{Acknowledgments}

We are grateful to Sangmi Chung for the pCBA-hrGFP expression plasmid and Jun Wei for helpful discussion. We also thank Mrs Xiu-Lan Li in our lab for her technical supports.

\section{References}

1 Strickland S, Mahdavi V. The induction of differentiation in teratocarcinoma stem cells by retinoic acid. Cell 1978; 15:393403.

2 Strickland S, Smith KK, Marotti KR. Hormonal induction of differentiation in teratocarcinoma stem cells: generation of parietal endoderm by retinoic acid and dibutyryl cAMP. Cell 1980; 21:347-355.

3 Kemler R, Brulet P, Schnebelen MT, Gaillard J, Jacob F. Reactivity of monoclonal antibodies against intermediate filament proteins during embryonic development. J Embryol Exp Morphol $1981 ; 64: 45-60$
4 Morita T, Tondella ML, Takemoto Y, et al. Nucleotide sequence of mouse EndoA cytokeratin cDNA reveals polypeptide characteristics of the type-II keratin subfamily. Gene 1988; 68:109-117.

5 Weiler-Guettler H, Aird WC, Rayburn H, Husain M, Rosenberg RD. Developmentally regulated gene expression of thrombomodulin in postimplantation mouse embryos. Development 1996; 122:2271-2281.

6 Dziadek M, Adamson ED. Localization and synthesis of alphafetoprotein in post-implantation mouse embryos. J Embryol Exp Morphol 1978; 43:289-313.

7 Chambers I, Colby D, Robertson M, et al. Functional expression cloning of Nanog, a pluripotency sustaining factor in embryonic stem cells. Cell 2003; 113:643-655.

8 Mitsui K, Tokuzawa Y, Itoh $\mathrm{H}$, et al. The homeoprotein Nanog is required for maintenance of pluripotency in mouse epiblast and ES cells. Cell 2003; 113:631-642.

9 Hart AH, Hartley L, Ibrahim M, Robb L. Identification, cloning and expression analysis of the pluripotency promoting Nanog genes in mouse and human. Dev Dyn 2004; 230:187-198.

10 Yamaguchi S, Kimura H, Tada M, Nakatsuji N, Tada T. Nanog expression in mouse germ cell development. Gene Expr Patterns 2005; 5:639-646.

11 Hamazaki T, Oka M, Yamanaka S, Terada N. Aggregation of embryonic stem cells induces Nanog repression and primitive endoderm differentiation. J Cell Sci 2004; 117:5681-686.

12 Yoshida-Koide U, Matsuda T, Saikawa K, et al. Involvement of Ras in extraembryonic endoderm differentiation of embryonic stem cells. Biochem Biophys Res Commun 2004; 313:475481.

13 Niwa H, Masui S, Chambers I, Smith AG, Miyazaki J. Phenotypic complementation stablishes requirements for specific POU domain and generic transactivation function of Oct-3/4 in embryonic stem cells. Mol Cell Biol 2002; 22:1526-1536.

14 Pan G, Pei D. The stem cell pluripotency factor NANOG activates transcription with two unusually potent subdomains at its C terminus. J Biol Chem 2005; 280:1401-1407.

15 Pan G, Pei D. Identification of two distinct transactivation domains in the pluripotency sustaining Factor Nanog. Cell Res 2003; 13:499-502.

16 Veltmaat JM, Orelio CC, Ward-Van Oostwaard D, et al. Snail is an immediate early target gene of parathyroid hormone related peptide signaling in parietal endoderm formation. Int J Dev Biol 2000; 44:297-307.

17 Chung S, Andersson T, Sonntag KC, et al. Analysis of different promoter systems for efficient transgene expression in mouse embryonic stem cell lines. Stem Cells 2002; 20:139-145.

18 Fujikura J, Yamato E, Yonemura S, et al. Differentiation of embryonic stem cells is induced by GATA factors. Genes Dev 2002; 16:784-789.

$19 \mathrm{Du}$ ZW, Cong XQ, Yao Z. Forced expression of the Oct-4 gene influences differentiation of embryonic stem cells. Chinese Sci Bull 2001; 46:1446-1449.

20 Soprano DR, Soprano KJ, Wyatt ML, Goodman DS. Induction of the expression of retinol-binding protein and transthyretin in F9 embryonal carcinoma cells differentiated to embryoid bodies. J Biol Chem 1988; 263:17897-17900.

21 Hogan B, Beddington R, Costantini F, Lacy E. Manipulating the Mouse Embryo: A Laboratory Manual. 2nd Edition. New York: 
Cold Spring Harbour Laboratory Press, 1994.

22 Hyslop LA, Stojkovic M, Armstrong L, et al. Downregulation of Nanog induces differentiation of human embryonic stem cells to extraembryonic lineages. Stem Cells 2005; 23:1035-1043.

23 Zhang JY, Wang X, Chen B, et al. Expression of Nanog gene promotes NIH3T3 cell proliferation. Biochem Biophys Res Commun 2005; 338:1098-1102.

24 Niwa H, Miyazaki J, Smith AG. Quantitative expression of Oct3/4 defines differentiation, dedifferentiation or self-renewal of ES cells. Nat Genet 2000; 24:372-376.
25 Kumar RM, Murray HL, Jenner RG, et al. Core transcriptional regulatory circuitry in human embryonic stem cells. Cell 2005; 122:947-956.

26 Constantinescu S. Stemness, fusion and renewal of hematopoietic and embryonic stem cells. J Cell Mol Med 2003; 7:103-112.

27 Chambers I, Smith AG. Self-renewal of teratocarcinoma and embryonic stem cells. Oncogene 2004; 23:7150-160.

Edited by Duanqing Pei 